\title{
Correlação do reflexo de Blinking com o quadro clínico de pacientes neurológicos graves
}

\author{
Correlation of the Blinking reflex with clinical feature in severe \\ neurological patients
}

\author{
Anelisa Mancastroppi Faria ${ }^{1}$, Daniella Silva Oggiam¹, Juliana Valéria Leite², Telma Dagmar \\ Oberg $^{3}$
}

\section{RESUMO}

Objetivo: Este estudo teve como objetivo correlacionar a melhora do nível de consciência e a força da musculatura inspiratória com o aparecimento do reflexo de blinking e demonstrar através destes acontecimentos o valor prognóstico deste reflexo. Método: Participaram do estudo 8 pacientes com faixa etária entre 20 e 82 anos, portadores de patologias neurológicas submetidos à Ventilação Mecânica (VM). Uma vez ao dia em horários fixos, foram avaliados no início do atendimento fisioterapêutico: a consciência através da Escala de Coma de Glasgow (ECG) e Escala de Coma de Jouvet (que inclui o reflexo de blinking) e, após o término do atendimento, a força da musculatura inspiratória através da medida da Pressão Inspiratória máxima (PImáx). Resultados: Pode-se observar que todos os pacientes que apresentaram reflexo de blinking positivo saíram da VM, portanto houve uma correlação direta entre o aparecimento do reflexo de blinking e a retirada da VM, assim como não houve relação direta entre estes acontecimentos e o dia em que o paciente apresentou a maior Plmáx com relação ao nível de consciência. Conclusão: Pode-se concluir que o reflexo de blinking é um marcador clínico, fidedigno, prático, não muito utilizado em pacientes em ventilação mecânica invasiva com rebaixamento do nível de consciência, sendo de grande importância para o prognóstico destes pacientes.

\section{Unitermos: Reflexo de Piscar, Blinking, Consciência, Músculos da Respiração, Ventilação mecânica.}

Citação: Faria AM, Oggiam DS, Leite JV, Oberg TD, Honorato DC. Correlação do reflexo de Blinking com o quadro clínico de pacientes neurológicos graves. Rev Neurocienc 2007, 15(1):17-20.

\section{SUMMARY}

Objective: The aim of this study was to correlate the improvement of the conscience level and the inspiratory muscle force with the appearance of the blinking reflex and to demonstrate through these events the valuable prognostic of this reflex. Method: A group of 8 patients with average age 20-82 years with neurological pathology submitted to Mechanical Ventilation (MV) was studied. Once a day in fixed schedules, the conscience level, through Glasgow Coma Scale and Jouvet Coma Scale (including the blinking reflex), was evaluated in the beginning of the physical therapy attendance and the inspiratory muscle force, through the measure of Maximum Inspiratory Pressure (MIP), at the end after attendance. Results: We observed that all the patients that had presented positive blinking reflex had left the MV. A direct correlation was noticed between the appearance of the blinking reflex and the withdrawal of the MV. There was no relation between these events and the day when the patient presented the greater MIP with

Trabalho realizado na Universidade Estadual de Campinas - UNICAMP, Campinas, São Paulo

1.Especializanda, Curso de Especialização de Fisioterapia Aplicada a Neurologia Adulto da Universidade Estadual de Campinas - UNICAMP, Campinas, São Paulo.

2.Fisioterapeuta Mestre, Supervisora do Curso de Especialização de Fisioterapia Aplicada a Neurologia Adulto da Universidade Estadual de Campinas - UNICAMP, Campinas, São Paulo.

3.Fisioterapeuta Doutora, Docente do Curso de Especialização de Fisioterapia Aplicada a Neurologia Adulto da Universidade Estadual de Campinas - UNICAMP, Campinas, São Paulo. 
conscience level. Conclusion: The blinking reflex is a practical, trustworthy clinical marker, not very used in patients in MV with degradation of the conscience level, and being of great importance for patients prognostic.

\section{Keywords: Blinking reflex, Conscience, Respiratory Muscles, Respiration- Artificial.}

Citation: Faria AM, Oggiam DS, Leite JV, Oberg TD, Honorato DC. Correlation of the Blinking reflex with clinical feature in severe neurological patients. Rev Neurocienc 2007, 15(1):17-20.

\section{INTRODUÇÃO}

Na neurologia hospitalar, a avaliação constante de pacientes com lesões no Sistema Nervoso Central torna-se necessária para determinar a evolução do quadro clínico e a intervenção fisioterapêutica. No entanto é necessária a utilização de escalas e testes que estabeleçam um sólido sistema capaz de acompanhar a evolução do nível de consciência destes pacientes ${ }^{1}$.

O nível de consciência depende da integridade do córtex cerebral e de sua ativação através da Formação Reticular Ascendente (FRA) que se localiza na região ponto-mesencefálica no tronco encefálico. Para que se observe alterações no nível de consciência, há necessidade de lesão ou disfunção (sejam elas traumáticas ou espontãneas) de grandes extensões nestas áreas.

Para avaliação do nível de consciência, existem várias escalas, duas mais usadas são: a Escala de Coma de Glasgow (ECG) e a Escala de Coma de Jouvet (ECJ), ambas se complementam. A ECG avalia a reatividade através da abertura ocular, resposta motora e resposta verbal, e a ECJ avalia as funções corticais através da perceptividade e as funções do tronco encefálico através da reatividade específica, inespecífica e autônoma. Assim, nos rebaixamentos de consciência mais intensos a ECG permite medir flutuações mais acuradamente. Já em estados próximos do normal a ECJ tem maior possibilidade de avaliar flutuações no nível de consciência, pois analisa melhor a função cortical, ou seja a consciência².

Em pacientes com rebaixamento do nível de consciência a perceptividade geralmente pode estar alterada, porém é de extrema importância ser avaliada. A consciência pode também ser verificada pela presença ou não do reflexo de piscamento a ameaça (reflexo de blinking). Este reflexo consiste no piscamento dos olhos em resposta a estímulos visuais externos, sendo um marcador clínico fidedigno, prático, não invasivo e de grande facilidade para avaliação da função cortical.

A positividade do reflexo de blinking é um sinal favorável para o prognóstico do paciente ${ }^{3}$. Este reflexo não é considerado um reflexo primitivo, ou seja, é um reflexo que somente estará presente ao término da maturação do Sistema Nervoso Central, pois requer um processo cortical de ordem mais alta. Portanto deve ser distinguido dos reflexos de tronco, como por exemplo, o reflexo corneopalpebral ${ }^{4}$.
Estudos acreditam que o reflexo de blinking é mediado corticalmente e requer o córtex visual primário intacto e mecanismos de ordem mais corticais, relacionados a atenção visual localizados nos campos oculares frontais e lóbulo parietal inferior ${ }^{4}$.

O dano cerebral pode ser acompanhado por alterações do sistema respiratório, pois a ventilação pulmonar depende da harmonia que envolve o Sistema Nervoso Central, caixa torácica, músculos respiratórios, vias aéreas e pulmões. Como conseqüência, uma alteração do padrão respiratório do paciente poderá ocasionar uma desproporção na concentração de $\mathrm{O}_{2}$ e $\mathrm{CO}_{2}$ que implicará em comprometimentos da difusão e perfusão sangüínea, prejudicando assim a oxigenação dos tecidos, principalmente pulmonar e cerebral. Por este motivo os indivíduos muitas vezes requerem suporte ventilatório, sendo submetidos à ventilação mecânica invasiva ${ }^{5}$

A mensuração e verificação de possível comprometimento na força da musculatura inspiratória é identificada pela pressão inspiratória máxima (PImáx), sendo esta mensurada pelo o manuvacuômetro ${ }^{6}$. No caso de pacientes sob ventilação mecânica invasiva com rebaixamento do nível de consciência a PImáx é mensurada utilizando uma válvula unidirecional acoplada ao manovacuômetro, que permite seletivamente a expiração enquanto a inspiração é bloqueada, com isso ocorre a iniciativa de sucessivos esforços com volumes respiratórios cada vez menores até atingir o volume residual, sendo interpretado como a PImáx do paciente?

Este trabalho teve como objetivo correlacionar o aparecimento do reflexo de blinking com as alterações do nível de consciência refletidas nos scores da ECG e ECJ, assim como possíveis alterações na força da musculatura inspiratória, avalidas pela PImáx, e na retirada do paciente da ventilação mecânica invasiva, e demonstrar sua importância como marcador clínico na evolução e prognóstico destes pacientes.

\section{MÉTODO}

No período de Setembro de 2005 a Janeiro de 2006 foram estudados indivíduos com lesões do Sistema Nervoso Central, apresentando rebaixamento do nível de consciência e submetidos à ventilação mecânica invasiva nas Enfermarias de Neuroclínica e Neurocirurgia 
do Hospital de Clínicas da Universidade Estadual de Campinas - UNICAMP. Para participar deste estudo os responsáveis assinaram um termo de consentimento Livre e Esclarecido conforme as exigências da resolução CNS - MS - 196/96, protocolo 678/2005, aprovado pelo Comitê de Ética em Pesquisa da Faculdade de Ciências Médicas - UNICAMP.

Foram incluídos no estudo, indivíduos de ambos os sexos com idade entre 20 a 85 anos, portadores de patologias neurológicas primárias, submetidos à ventilação mecânica invasiva (ventilados por tubo orotraqueal ou por traqueostomia), que apresentassem drive respiratório e estivessem hemodinâmicamente estável, sem sedação e que tivessem indicação de atendimento fisioterapêutico. Foram excluídos do estudo indivíduos com hipótese diagnóstica de morte encefálica e sem indicação de tratamento fisioterapêutico.

\section{Procedimento}

Inicialmente foram colhidos os sinais vitais (pressão arterial, freqüência cardíaca, freqüência respiratória e temperatura corporal) para certificação da estabilidade hemodinâmica (critério de inclusão).

Os pacientes eram mantidos em decúbito dorsal e submetidos à avaliação do nível de consciência a partir da Escala de Coma de Glasgow e Escala de Coma de Jouvet incluindo o reflexo de blinking.

A fisioterapia convencional foi mantida e esta incluía a fisioterapia motora que consiste em alongamentos, mobilizações passivas e posicionamento e a fisioterapia respiratória que consiste em manobras de higiene brônquica, reexpansão pulmonar, aspiração e condutas para desmame da ventilação mecânica invasiva. Imediatamente após o término da terapia foi realizada a mensuração da PImáx, desconectando o paciente da ventilação mecânica invasiva, acoplando a válvula unidirecional com o manuvacuômetro no tubo orotraqueal ou traqueostomia, permanecendo por trinta segundos, conectando o paciente novamente ao ventilador mecânico e ventilando-o por um minuto entre uma mensuração e outra, sendo considerado o maior valor obtido da PImáx em três medidas consecutivas.

O estudo foi realizado uma vez ao dia até a retirada do paciente da ventilação mecânica invasiva ou até apresentar melhora do nível de consciência expressada pela positividade do reflexo de blinking associada a uma resposta motora coerente a comandos verbais (resposta motora 6 na ECG).

\section{RESULTADOS}

Foram estudados 8 pacientes com diferentes diagnósticos de patologias neurológicas (Traumatismo cra- niano, tumor cerebrai, Acidente vascular cerebral) sendo que $50 \%$ dos pacientes saíram da ventilação mecânica, 37,5\% foram a óbito e 12,5\% foram encaminhados para a unidade de terapia intensiva (UTI).

Na Tabela 1 observa-se a permanência dos pacientes em ventilação mecânica invasiva durante o período de internação nas enfermarias, correlacionando o dia em que o paciente apresentou o reflexo de blinking e o dia em que ele apresentou a maior PImáx com a permanência na ventilação mecânica. Sendo que os pacientes 5, 7 e 8 foram a óbito e o 3 foi encaminhado para UTI.

Tabela 1. Dados referentes ao dia que apresentou reflexo de blinking, retirada da Ventilação Mecânica (VM) e máximo valor da Pressão Inspiratória máxima (Pimáx).

\begin{tabular}{|c|c|c|c|}
\hline Pacientes & $\begin{array}{c}\text { Dia que apresentou } \\
\text { blinking }\end{array}$ & $\begin{array}{c}\text { Último dia } \\
\text { de VM }\end{array}$ & $\begin{array}{c}\text { Dia que apresentou } \\
\text { valor máximo de } \\
\text { PImáx }\end{array}$ \\
\hline 1 & 9 & 9 & 6 \\
\hline 2 & 17 & 17 & 8 \\
\hline 3 & - & - & 6 \\
\hline 4 & 3 & 3 & 2 \\
\hline 5 & - & - & 2 \\
\hline 6 & 11 & 11 & 6 \\
\hline 7 & - & - & 4 \\
\hline 8 & - & - & 3 \\
\hline
\end{tabular}

Os Gráficos 1 e 2 são relativos aos pacientes que sobreviveram. No gráfico 1 observa-se os scores da Escala de Coma de Glasgow, referente ao primeiro dia e ao ultimo dia de permanência na ventilação mecânica. O gráfico 2 demonstra o score da Escala de Coma de Jouvet, lembrando que ao contrário da ECG, quanto menor o score da ECJ melhor o nível de consciência do paciente.

O Gráfico 3 apresenta a PImáx inicial (primeiro dia de mensuração) e a PImáx final (último dia de mensuração) demonstrando a evolução na força da musculatura inspiratória dos pacientes.

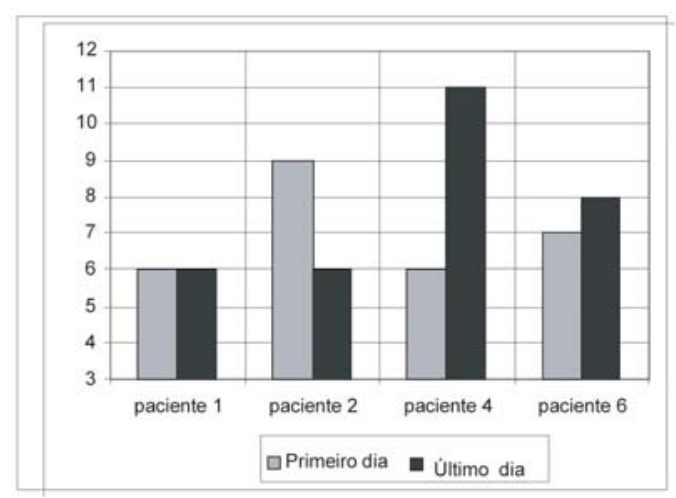

Gráfico 1. Evolução do nível de consciência de acordo com a ECG durante a permanência em Ventilação Mecânica 


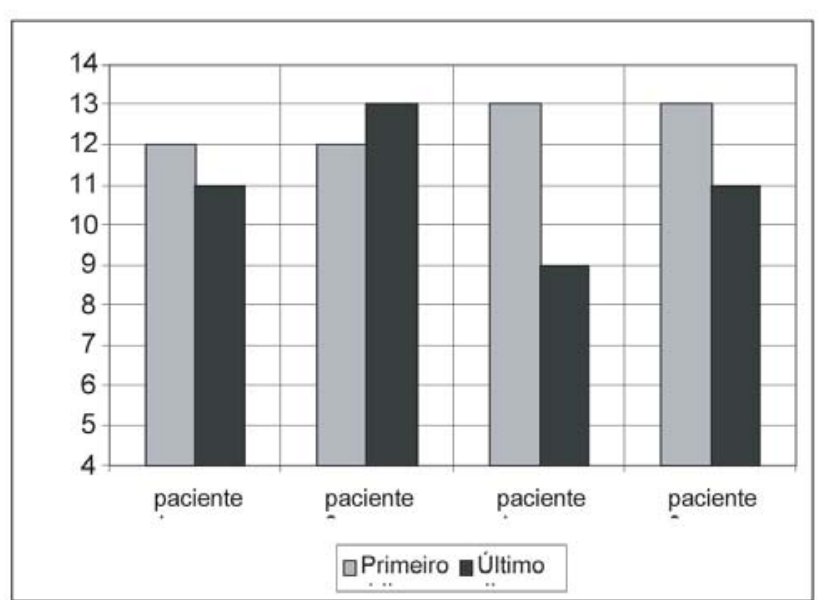

Gráfico 2. Evolução do nível de consciência de acordo com a ECJ durante a permanência em Ventilação Mecânica

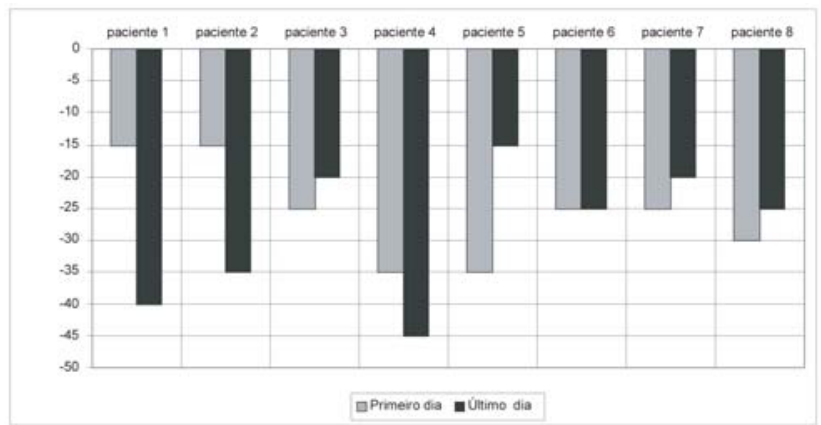

Gráfico 3. Evolução da Pressão inspiratória máxima (PImáx) durante a permanência em ventilação .

\section{DISCUSSÃO}

Após este estudo observou-se que os resultados obtidos dependem diretamente da condição clínica de cada individuo, uma vez que os dados foram colhidos de pacientes portadores de variadas patologias neurológicas.

Através da análise dos dados obtidos pode-se observar uma correlação direta entre o aparecimento do reflexo de blinking com a retirada do paciente da ventilação mecânica, e não houve uma relação direta entre o dia em que os pacientes apresentaram a maior PImáx e o dia em que foi retirado a ventilação mecânica. Foi observado também que os pacientes que não apresentaram o reflexo de blinking, não saíram da ven-

\section{REFERÊNCIAS BIBLIOGRÁFICAS}

1. Muniz ECS, Thomaz MCA, Kubota MY, Cianci L, Sousa RMC. Utilização da escala de coma de Glasgow e escala de coma de Jouvet para avaliação do nível de consciência. Rev Esc Enf USP 1997; 31(2): 287-303.

2. Gattaz MD. Depressão da consciência. Rev Psiquiatr Clin 1997; 24(4):1824.

3. Rumpl E, Gerstenbrand F, Hack JM, Prugger M. Some observations on the blink reflex in posttraumatic coma. Electroencephalogr Clin Neurophysiol. 1982; 54(4): 406-417.

4. Liu GT, Ronthal M. Reflex Blink to Visual Threat. J Clin Neuro Ophthalmol tilação mecânica, evoluindo a óbito ou encaminhados para UTI.

Comparando os resultados dos gráficos 1 e 2, com a tabela já citada, podemos concluir que não há uma relação direta entre a retirada da ventilação mecânica, o aparecimento do reflexo de blinking e o dia da maior PImáx com a melhora do nível de consciência refletidos nos scores das escalas de coma.

Os pacientes 1, 2, 4 e 6 apresentaram o reflexo de Blinking associado a retirada da ventilação mecânica mesmo estando com scores nas escalas de coma inferiores aos scores de sua admissão.

Relacionando o primeiro dia de mensuração da PImáx com o último dia, de acordo com o gráfico 3, pode-se observar que os pacientes que apresentaram o reflexo de Blinking e saíram da ventilação mecânica apresentaram melhora ou estabilização da PImáx, e os pacientes que foram a óbito ou encaminhados para UTI apresentaram uma involução da PImáx, podendo ser justificado por deteriorização da força muscular inspiratória, causada pela gravidade da lesão cerebral ou por tempo prolongado em ventilação mecânica invasiva.

Foi observado que os pacientes 1, 2, 4 e 6, apresentaram melhora da força muscular inspiratória e reflexo de blinking positivo, saíram da ventilação mecânica, sendo que estes não apresentaram o reflexo de blinking no dia de maior PImáx dentre o período de permanência na ventilação mecânica demonstrando que não há uma relação direta com saída da VM e dia em que apresentou maior PImáx, e sim com o reflexo de blinking.

Dos pacientes que não apresentaram blinking, diminuíram a PImáx e não saíram da ventilação mecânica, sendo estes 5,7 e 8 que evoluíram a óbito e o 3 foi encaminhado para UTI.

A partir destes resultados, pode-se concluir que o reflexo de blinking é um marcador clínico fidedigno e prático, pouco utilizado em pacientes em ventilação mecânica invasiva com rebaixamento do nível de consciência, sendo de grande importância para o prognóstico destes pacientes, e merece maior atenção na prática clínica assim como mais pesquisas como, que visem, por exemplo, uma continuidade na avaliação dos pacientes até a alta hospitalar para acompanhar a evolução do quadro destes de maneira mais prolongada.
1992; 12(1): 47-56

5. Adams RD, Victor M, Ropper AH. Principles of Neurology. 60 ed. SantiagoChile: The McGraww-Hill; 1998

6. Caruso P, Friedrich C, Denari SDC, Ruiz SAL, Deheinzelin D. The Unidirectional Valve Is the Best Method To Determine Maximal Inspiratory Pressure During Weaning. Clin Invest Crit Care 1999; 115:1096-1101.

7. Monteiro LS, Veloso CA, Araújo S, Figueiredo LC, Terzi RGG. Comparision of Two Methods of Measurement of Maximal Inspiratory Pressure with Unidirectional Valve. Rev Bras Ter Inten 2004; 16(2):74-77. 